\title{
Wounding-Induced WRKY8 Is Involved in Basal Defense in Arabidopsis
}

\author{
Ligang Chen, ${ }^{1,2}$ Liping Zhang, ${ }^{1,2}$ and Diqiu $\mathrm{Yu}^{1}$ \\ ${ }^{1}$ Key Laboratory of Tropical Forest Ecology, Xishuangbanna Tropical Botanical Garden, Chinese Academy of Sciences, \\ Mengla, Yunnan 666303, China; ${ }^{2}$ The Graduate School of the Chinese Academy of Sciences, Beijing 100049, China
}

Submitted 6 July 2009. Accepted 5 January 2010.

\begin{abstract}
The WRKY family of plant transcription factors controls several types of plant stress responses. Arabidopsis WRKY8, localized to the nucleus, is mainly induced by abscissic acid, $\mathrm{H}_{2} \mathrm{O}_{2}$, wounding, Pseudomonas syringae and Botrytis cinerea infection, and aphid and maggot feeding. To determine its biological functions, we isolated loss-offunction T-DNA insertion mutants and generated gain-offunction overexpressing WRKY 8 transgenic plants in Arabidopsis. Plants expressing the mutated WRKY8 gene showed increased resistance to $P$. syringae but slightly decreased resistance to $B$. cinerea. In contrast, transgenic plants overexpressing $W R K Y 8$ were more susceptible to $P$. syringae infection but more resistant to $B$. cinerea infection. The contrasting responses to the two pathogens were correlated with opposite effects on pathogen-induced expression of two genes; salicylic acid-regulated $P A T H O$ GENESIS-RELATED1 (PRI) and jasmonic acid-regulated $P D F 1.2$. Therefore, our results suggest that $W R K Y 8$ is a negative regulator of basal resistance to $P$. syringae and positive regulator to $B$. cinerea.
\end{abstract}

During the long-term and constant interactions with various microbial pathogens or herbivores, resistant plants have successfully evolved sophisticated defense mechanisms to protect themselves. First, resistant plants recognize microbes or pathogen-associated molecular patterns (PAMP) via pattern recognition receptors, and then activate basal defense responses to halt pathogen infection. This is known as PAMP-triggered immunity (Jones and Dangl 2006). Second, plant hosts recognize specific effector molecules that are introduced into the plant by the pathogen, and then produce disease resistance proteins that activate highly efficient effector-triggered immunity (Jones and Dangl 2006).

Salicylic acid (SA), ethylene (ET), and jasmonic acid (JA) are the main signaling molecules involved in defense responses. The SA signaling pathway is mainly linked to resistance to biotrophic pathogens. The JA and ET signaling pathways me-

Ligang Chen and Liping Zhang contributed equally to this work.

Nucleotide sequence data for the genes described in this article are available from The Arabidopsis Genome Initiative database under the following accession numbers: WRKY8 (AT5G46350), PRI (At2g14610), and PDF1.2 (At5G44420).

Corresponding author: Diqiu Yu; Telephone: +86.871.5178133; Fax: +86.871.5160916; E-mail: ydq@xtbg.ac.cn

* The $\boldsymbol{e}$-Xtra logo stands for "electronic extra" and indicates that three supplementary figures are available online and that Figure 5 appears in color online. diate resistance mainly to necrotrophic pathogens. Thus, there are complicated defense networks that are induced in response to different types of invading pathogens. Interestingly, the SA and ET/JA signaling pathways often interact in an antagonistic manner (Kunkel and Brooks 2002). For instance, disruptions of JA signaling regulators such as COI1 result in enhanced SA accumulation and signaling in pathogen-infected plants (Kloek et al. 2001), while blocking SA accumulation can promote JA signaling (Spoel et al. 2003). Besides SA, ET, and JA, abscissic acid (ABA) also plays negative or positive roles in plant responses to pathogen attack. For example, enhanced ABA levels are associated with increased susceptibility, whereas reduced ABA levels can increase resistance to many pathogens (Asselbergh et al. 2008; Mauch-Mani and Mauch 2005). Importantly, ABA also has a positive effect on virus infection by inhibiting the transcriptional level of a basic $\beta-1,3-$ glucanase (PR2) (Rezzonico et al. 1998).

In Arabidopsis, the plant WRKY transcription superfamily consists of an estimated 74 members that fall into three major structural groups (Eulgem 2005). There is a large body of evidence demonstrating that specific WRKY proteins can function as positive or negative regulators in the basal defense responses of plants. For example, mutations in Arabidopsis WRKY7O enhanced susceptibility to both biotrophic and necrotrophic pathogens, including the bacterial pathogen Erwinia carotovora and the fungal pathogens Erysiphe cichoracearum and Botrytis cinerea (Li et al. 2004, 2006; AbuQamar et al. 2006). In addition, wrky70 mutants are compromised in both basal defense and full RPP4-mediated disease resistance to the oomycete Hyaloperonospora parasitica (Knoth et al. 2007). Disruption of WRKY33 enhanced susceptibility to the necrotrophic fungal pathogens $B$. cinerea and Alternaria brassicicola (Zheng et al. 2006). Virus-induced silencing of three $W R K Y$ genes in tobacco compromised $N$-gene-mediated resistance to Tobacco mosaic virus (Liu et al. 2004). Overexpression of OsWRKY23 or OsWRKY45 in Arabidopsis conferred enhanced disease resistance against the bacterial pathogen Pseudomonas syringae (Jing et al. 2009; Qiu and Yu 2009). Thus, certain pathogen-induced WRKY proteins can positively regulate plant disease resistance.

In contrast, several recent studies have shown that a number of WRKY proteins can function as negative regulators of basal defense responses in plants. For example, mutations of Arabidopsis WRKY7, WRKY11, and WRKY17 enhance basal resistance to virulent $P$. syringae strains (Park et al. 2005; JournotCatalino et al. 2006; Kim et al. 2006). Likewise, the structurally related WRKY18, WRKY40, and WRKY60 also have partially redundant functions as negative regulators of plant resistance against the biotrophic bacterial pathogen $P$. syringae and the fungal pathogen E. cichoracearum (Xu et al. 2006; 
Shen et al. 2007). Recently, WRKY48 and two structurally related proteins, WRKY38 and WRKY62, were shown to have additive functions as negative regulators of basal defense against the bacterial pathogen $P$. syringae (Kim et al. 2008; Xing et al. 2008). As well, barley HvWRKY1 and HvWRKY2 function as PAMP-inducible suppressors of basal defense (Shen et al. 2007). Thus, we can deduce that WRKY transcription factors act as both positive and negative regulators, and that they participate in the tight regulation and fine-tuning of the complex signaling and transcriptional networks of plant defense.

Previous study has shown that numerous $W R K Y$ genes were induced by $P$. syringae infection at early stages (Dong et al. 2003). However, it is unclear whether WRKY genes' expression was affected by $P$. syringae infection at later stages. Based on the obviously stronger expression of WRKY 8 in the wild type 2 days after $P$. syringae infection, we choose WRKY 8 for further analysis. Here, we show that Arabidopsis WRKY8, which is localized in the nucleus, is induced by mechanical wounding, aphid and maggot feeding, ABA treatment, and pathogen infection. To directly determine the biological functions of WRKY8, we have isolated loss-of-function T-DNA insertion mutants and generated gain-of-function transgenic plants overexpressing WRKY8. Functional analysis of wrky 8 mutants and transgenic $35 S$ :WRKY 8 plants for response to microbial pathogens indicated that the pathogeninduced WRKY 8 transcription factor plays important roles in plant disease resistance.

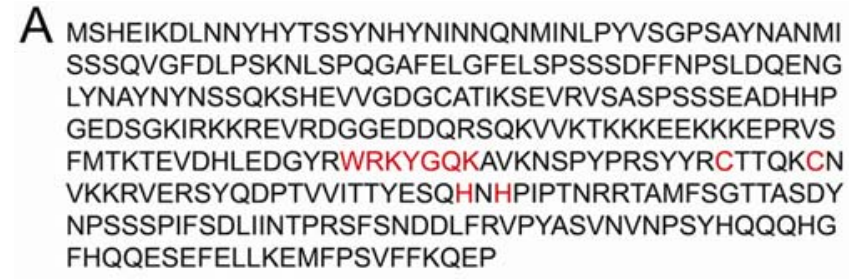

B 35S:WRKY8:GFP

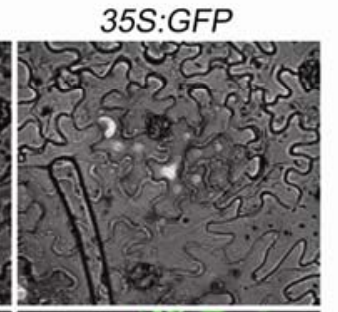
35S:GFP
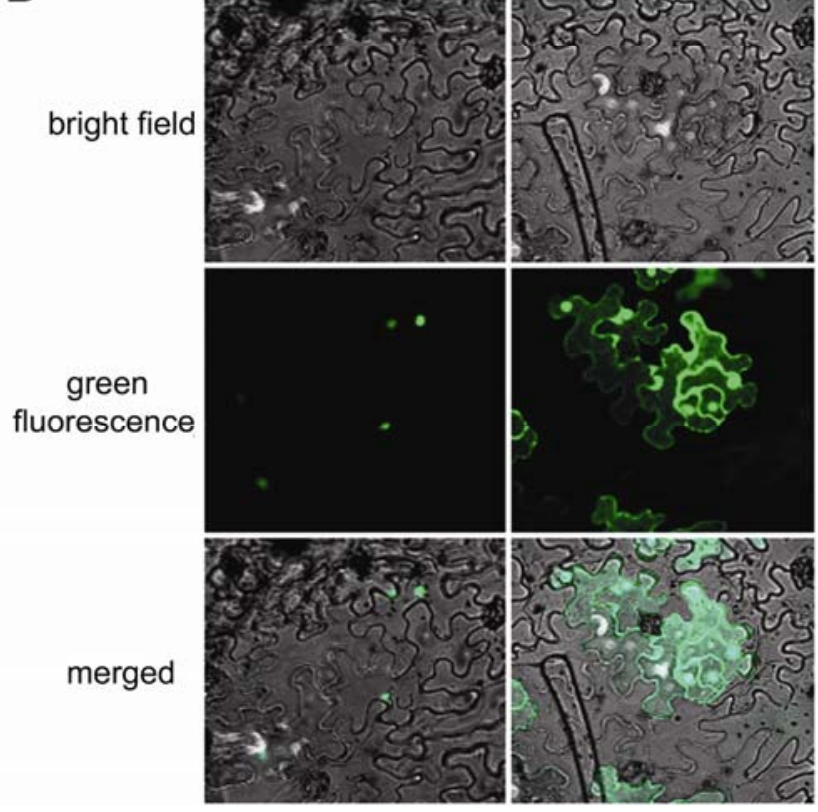

Fig. 1. Sequence and subcellular localization of WRKY8. A, Amino acid sequence of WRKY8. Highly conserved WRKYGQK sequences and residues forming $\mathrm{C}_{2} \mathrm{H}_{2}$ zinc-fingers are shown in red. B, Subcellular localization of WRKY8. WRKY8 was fused to green fluorescent protein (GFP) to yield WRKY8:GFP. The chimeric protein was localized to the nucleus of Nicotiana benthamiana leaf cells (left) whereas GFP alone was detected in both the nucleus and the cytoplasm due to its small size (right).

\section{RESULTS}

Protein structure, subcellular localization, and expression pattern.

AtWRKY8 (AT5G46350) encodes a protein of 326 amino acids with a molecular weight of $37293.8 \mathrm{Da}$ and an isoelectric point of 8.0888 (Fig. 1A). Sequence analyses revealed that the WRKY8 protein contains one DNA-binding domain (WRKY domain) located at amino acid positions 182 to 241, and is classified as a group II WRKY protein (Eulgem et al. 2000). As a putative transcription factor, WRKY8 is likely to be localized in the nucleus. To determine the subcellular localization of the WRKY8 protein, we fused the full-length WRKY8 cDNA to the green fluorescent protein (GFP) gene, then infiltrated the construct into Nicotiana benthamiana leaves via Agrobacterium spp. infiltration. We analyzed transient expression of the construct using reverse-transcription polymerase chain reaction (RT-PCR) (data not shown). The transiently expressed $p W R K Y 8: G F P$ fusion protein was localized exclusively to the nucleus (Fig. 1B). In contrast, free GFP was found in both the nucleus and cytoplasm (Fig. 1B). These results indicate that $W R K Y 8$ protein is localized to the nucleus, supporting its predicted role as a transcriptional regulator.

Next, we examined the expression pattern of WRKY8 in Arabidopsis. First, we used RNA gel blot analyses to investi-

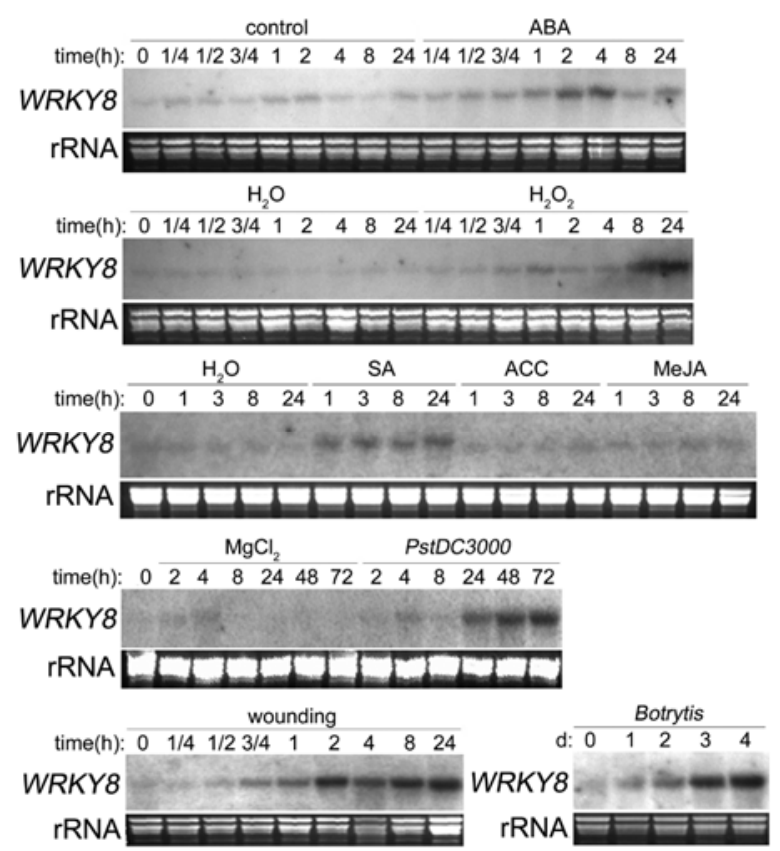

Fig. 2. Expression of WRKY8. RNA samples were prepared from 4-weekold Arabidopsis plants (Col-0) at given times (hours or days) after spraying with $\mathrm{H}_{2} \mathrm{O}$, abscisic acid (ABA) $(0.1 \mathrm{mM})$, jasmonic acid (JA) (0.1 $\mathrm{mM})$, ethylene (ET) $(0.1 \mathrm{mM})$, salicylic acid (SA) $(1 \mathrm{mM})$, or $\mathrm{H}_{2} \mathrm{O}_{2}(5$ $\mathrm{mM})$. Isolated RNAs were probed with a WRKY8 cDNA fragment. Ethidium bromide-stained ribosomal RNA was used as a loading control. For wounding treatments, leaves of 4-week-old plants were squeezed with forceps two or three times per leaf (approximately 50\% of the leaf area) and RNA was isolated from wounded leaves at indicated times. Northern blot analyses were carried out to reveal expression of AtWRKY8. For Pseudomonas syringae pv. tomato DC3000 treatment, 5-week-old wild-type (Col-0) plants were infiltrated with a suspension of $P$. syringae pv. tomato DC3000 (optical density at $600 \mathrm{~nm}=0.0001$ in $10 \mathrm{mM} \mathrm{MgCl}$ ). Inoculated leaves were collected at indicated times, then RNAs were isolated and Northern blot analyses were carried out. For Botrytis spp. treatment, 5-week-old wild-type plants were inoculated by spraying with a spore suspension $\left(5 \times 10^{4}\right.$ spores $\left./ \mathrm{ml}\right)$. Plants were maintained under high humidity and whole seedlings were collected for isolation of RNA at indicated times. Expression of WRKY 8 was determined by Northern blot analysis. 
gate expression of the $W R K Y 8$ gene in response to various treatments. Expression of WRKY 8 was rapidly induced by ABA treatment $(100 \mu \mathrm{M})$ and peaked at $4 \mathrm{~h}$ (Fig. 2), which is consistent with the existence of ABRE elements upstream the WRKY8 promoter (Supplementary Fig. 1). Expression of WRKY8 was also induced by $\mathrm{H}_{2} \mathrm{O}_{2}$. Expression of WRKY8 was slightly induced by SA but not by methyl jasmonate (MeJA) or 1-aminocyclopropane-1-carboxylate (ACC) treatments (Figs. 2 and $3 \mathrm{Ai}$ ). We also examined expression of the WRKY8 gene during biotic and abiotic stress treatments. Expression of WRKY 8 was strongly induced in P. syringae-infected plants at 1,2 , and 3 days postinoculation (dpi), compared with $\mathrm{MgCl}_{2-}$ infiltrated plants (Fig. 2). Infection of $B$. cinerea also induced strongly expression of WRKY8 (Fig. 2). Accumulation of WRKY8 messenger RNA (mRNA) was detected as early as 2 dpi and levels of expression remained high during the infection of $B$. cinerea. Mechanical wounding also induced expression of WRKY8. Together, these results indicate that the WRKY 8 gene is involved in responses to multiple stresses.

To determine the expression patterns of WRKY 8 more precisely, we generated two homozygous $\mathrm{T}_{3}$ lines of $p W R K Y 8$ : GUS transgenic plants. Once we confirmed that WRKY8 was upregulated by $\mathrm{ABA}, \mathrm{H}_{2} \mathrm{O}_{2}, B$. cinerea, $P$. syringae, and wounding by Northern blotting analysis (Fig. 2), we next tested whether the WRKY8 promoter was responsible for these upregulations. $\beta$-glucuronidase (GUS) activities were observed in leaves after $\mathrm{ABA}, \mathrm{H}_{2} \mathrm{O}_{2}$, wounding or rubbing treatments, and B. cinerea infection (Fig. $3 \mathrm{~A}$ ). Interestingly, AtWRKY8 was also expressed in response to aphid or maggot feeding because $G U S$ expression was observed at the sites of feeding (Fig. 3Ae and f). In addition to the abovementioned pattern of GUS activity, the infection of $P$. syringae induced more strongly GUS expression in infected plants than in $\mathrm{MgCl}_{2}$-infiltrated plants, especially at 2 and 3 dpi (Fig. 3B).
Together, the Northern blot and GUS staining results show that WRKY8 is mainly induced by $\mathrm{SA}, \mathrm{ABA}, \mathrm{H}_{2} \mathrm{O}_{2}$, wounding, $P$. syringae and $B$. cinerea infection, and aphid and maggot feeding.

To determine whether wounding- and pathogen-induced WRKY8 expression is influenced by the SA, ET, or JA signaling pathways, wounding- and pathogen-induced WRKY 8 expression was monitored in various signaling mutants. Induced WRKY8 expression was modestly reduced in the nprl-3 and sid2-3 mutants, which are defective in SA signaling and biosynthesis, respectively (Cao et al. 1997; Wildermuth et al. 2001) (Fig. 4). By contrast, in the JA-insensitive coil-1 and ET-insensitive ein2-1 mutants (Xie et al. 1998; Alonso et al. 1999), there were slightly higher expression levels of WRKY8 transcripts than those in wild-type plants (Fig. 4). These results suggest that wounding- and pathogen-induced WRKY8 expression is positively regulated by the SA signaling pathway but negatively regulated by the JA and ET signaling pathways.

\section{Isolation of three AtWRKY8 T-DNA insertion mutants.}

To study the functions of WRKY 8 directly, we first characterized three T-DNA insertion alleles of WRKY8 from Salk TDNA populations (Alonso et al. 2003). The T-DNA insertions in the mutants, designated wrky8-1 (SALK_107668), wrky8-2 (SALK_050194), and wrky8-3 (SALK_117175), were first confirmed by PCR using primers specific to the WRKY 8 gene and the T-DNA insertions in the three alleles (data not shown). The three wrky 8 mutant alleles carry T-DNA insertions in the first intron ( $720 \mathrm{bp}$ from the translation start), the second intron $(2,123 \mathrm{bp}$ from the translation start), and the third exon $(2,347$ bp from the translation start), respectively (Fig. 5A). Northern blotting and RT-PCR analysis confirmed the absence of the normal, full-length WRKY8 transcripts in wounding-treated wrky $8-1$ homozygous lines, and a very faint band in the wrky $8-2$
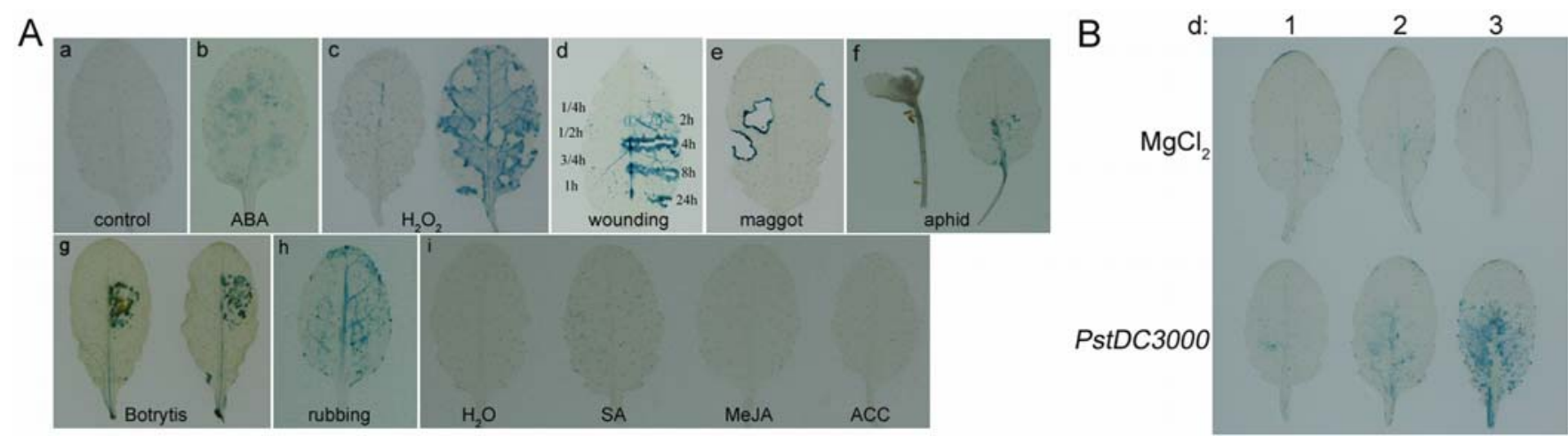

Fig. 3. $\beta$-Glucuronidase (GUS) staining of WRKY8. A, GUS staining of AtWRKY8 after treatment with abscisic acid $(A B A), \mathrm{H}_{2} \mathrm{O}_{2}$, wounding, maggot or aphid feeding, Botrytis infection; $\mathrm{Aa}, \mathrm{H}_{2} \mathrm{O}$; Ab, ABA (4 h); Ac, $\mathrm{H}_{2} \mathrm{O}_{2}$ (left, 4 h; right 24 h); Ad, wounding (time course of 24, 8, 4, 2, 1, three-quarter, half, and one-quarter h, respectively); Ae, maggot feeding; Af, aphid feeding; Ag, Botrytis infection(3 days); Ah, rubbing (24 h); Ai, salicylic acid (SA), methyl jasmonate (MeJA), and 1-aminocyclopropane-1-carboxylate (ACC) $(3 \mathrm{~h})$. B, GUS staining of AtWRKY8 at indicated times after inoculation with $10 \mathrm{mM}$ $\mathrm{MgCl}_{2}$ or Pseudomonas syringae pv. tomato DC3000 (optical density at $600 \mathrm{~nm}=0.0001$ in $10 \mathrm{mM} \mathrm{MgCl} 2$ ). These experiments were performed two times with similar results.

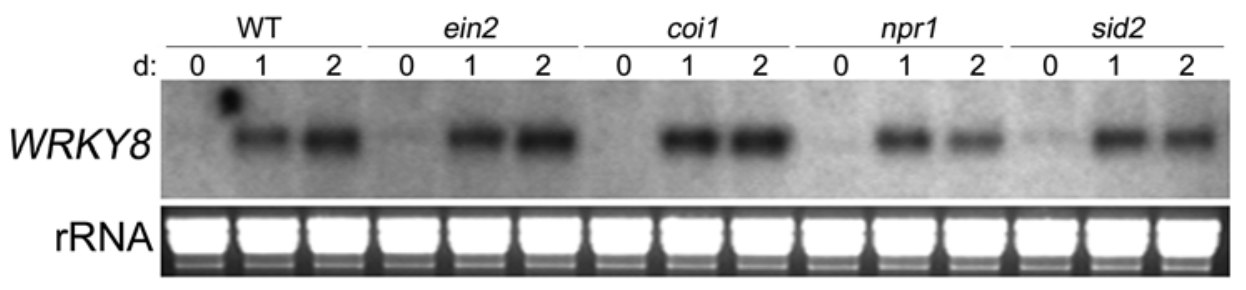

Fig. 4. Expression of WRKY8 in defense signaling mutants infected with Pseudomonas syringae pv. tomato DC3000. Five-week-old Arabidopsis wild-type (WT) $\left(\right.$ Col-0) and mutant plants were infiltrated with $P$. syringae pv. tomato DC3000 (optical density at $600 \mathrm{~nm}=0.0001$ in $10 \mathrm{mM} \mathrm{MgCl}_{2}$ ). The infiltrated leaves were collected at indicated times after inoculation for RNA isolation. Northern blot analyses were carried out to reveal expression of AtWRKY8. 
and wrky8-3 mutant plants (Fig. 5B; Supplementary Fig. 2).The wrky 8 mutants grew at the same rate and flowered at the same time as wild-type plants, and showed normal plant morphology.

To characterize the role of WRKY 8 more precisely, we generated transgenic Arabidopsis plants constitutively expressing WRKY8 under the control of the Cauliflower mosaic virus (CaMV) 35S promoter. Northern blotting showed that several transgenic plants constitutively expressed high levels of WRKY 8 transcripts, even in the absence of pathogen infection or wounding treatments. Similar to transgenic Arabidopsis plants that constitutively express WRKY18 or WRKY48 (Chen and Chen 2002; Xing et al. 2008), plants constitutively expressing WRKY 8 were smaller in size and had slightly serrated leaves compared with the wild type (Fig. 5D). Finally, we chose two transgenic lines (Fig. 5C, nos. 4 and 7) for further study. These lines constitutively expressed WRKY8 at elevated levels and contained a single T-DNA locus in their genomes based on the ratio of antibiotic resistance phenotypes.

\section{Disrupted or altered WRKY8 expression}

affected responses to $P$. syringae.

To determine the possible roles of the WRKY 8 gene in plant defense, we first examined the response of wild-type and

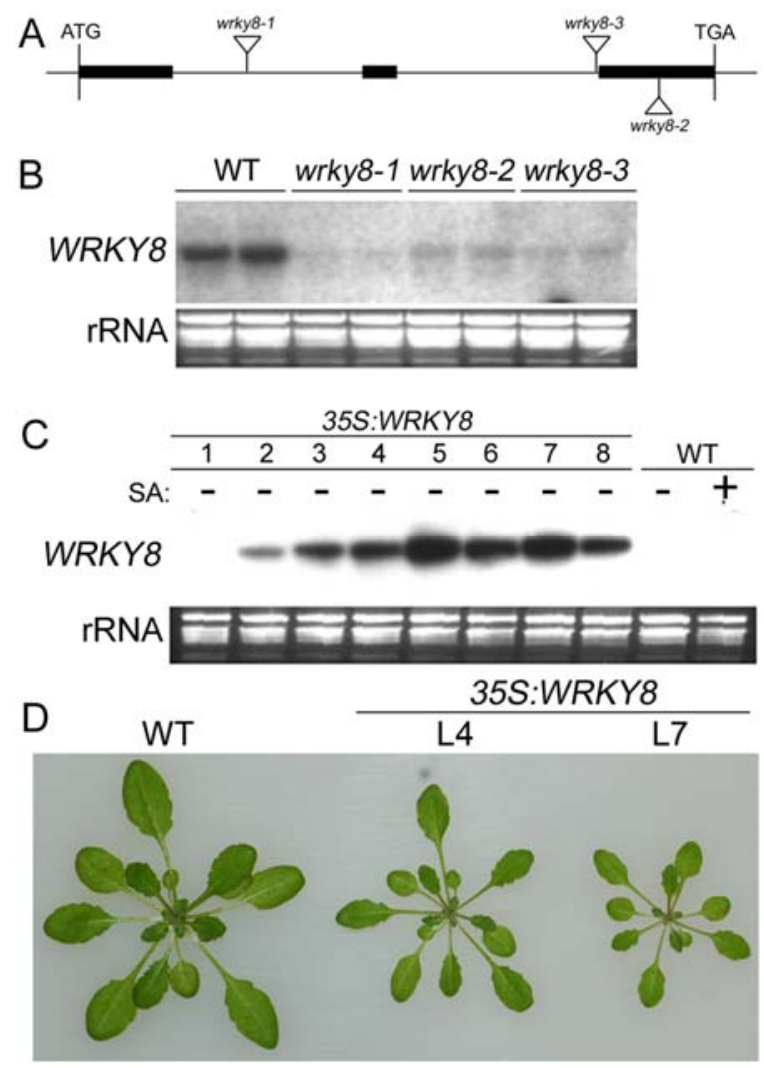

Fig. 5. T-DNA insertion mutants and overexpression lines for WRKY8. A, Diagram of $W R K Y 8$ gene and T-DNA insertions in AtWRKY 8 mutants. B, Northern blot analysis of wrky 8 mutants. RNA samples were prepared from 24-day-old wild-type (WT) Arabidopsis and wrky 8 mutant leaves $4 \mathrm{~h}$ after wounding treatment. RNAs were probed with a WRKY8 cDNA fragment. Ethidium bromide-stained ribosomal RNA (rRNA) was used as a loading control. C, Northern blot analysis of WRKY8 expression in transgenic plants constitutively overexpressing $W R K Y 8$. RNA samples were prepared from leaves of eight transgenic 35S:WRKY8 lines or leaves of WT plants (Col-0) (treated with $2 \mathrm{mM}$ salicylic acid or untreated). RNAs were probed with a WRKY 8 cDNA fragment, and ethidium bromide-stained rRNA was used as a loading control. D, Phenotype of representative 32-day-old WT (Col-0) and 35S:W8 lines 4 (L4) and 7 (L7). Both L4 and L7 contained a single TDNA insertion in the genome and stably expressed WRKY8. Homozygous F3 progeny plants were used in all experiments. wrky8 mutant plants to a virulent strain of $P$. syringae. Plants were inoculated with the bacteria and the growth of the pathogen was monitored. The homozygous wrky 8 mutants had an approximately two- to threefold reduction in the growth of the bacterial pathogen at $3 \mathrm{dpi}$, compared with the wild-type (Fig. 6A). Moreover, the inoculated leaves of the wrky8 mutant showed less chlorosis than wild-type plants at 3 dpi (Fig. 6B). Thus, disruption of WRKY 8 enhanced resistance to the bacterial pathogen.

To further characterize the role of $W R K Y 8$ in defense against $P$. syringae, we compared bacterial growth in $35 S: W R K Y 8$ transgenic plants with that in wild-type plants. Conversely to wrky 8 mutants, there was a marked increase (seven- to 10fold) in bacterial growth in $35 S: W R K Y 8$ transgenic plants compared with wild-type plants (Fig. 6A). The transgenic plants also developed more severe disease symptoms than the wild-type plants after infection with $P$. syringae (Fig. 6B). Thus, constitutive overexpression of WRKY 8 led to increased growth of the bacterial pathogen and enhanced development of disease symptoms in the transgenic plants.
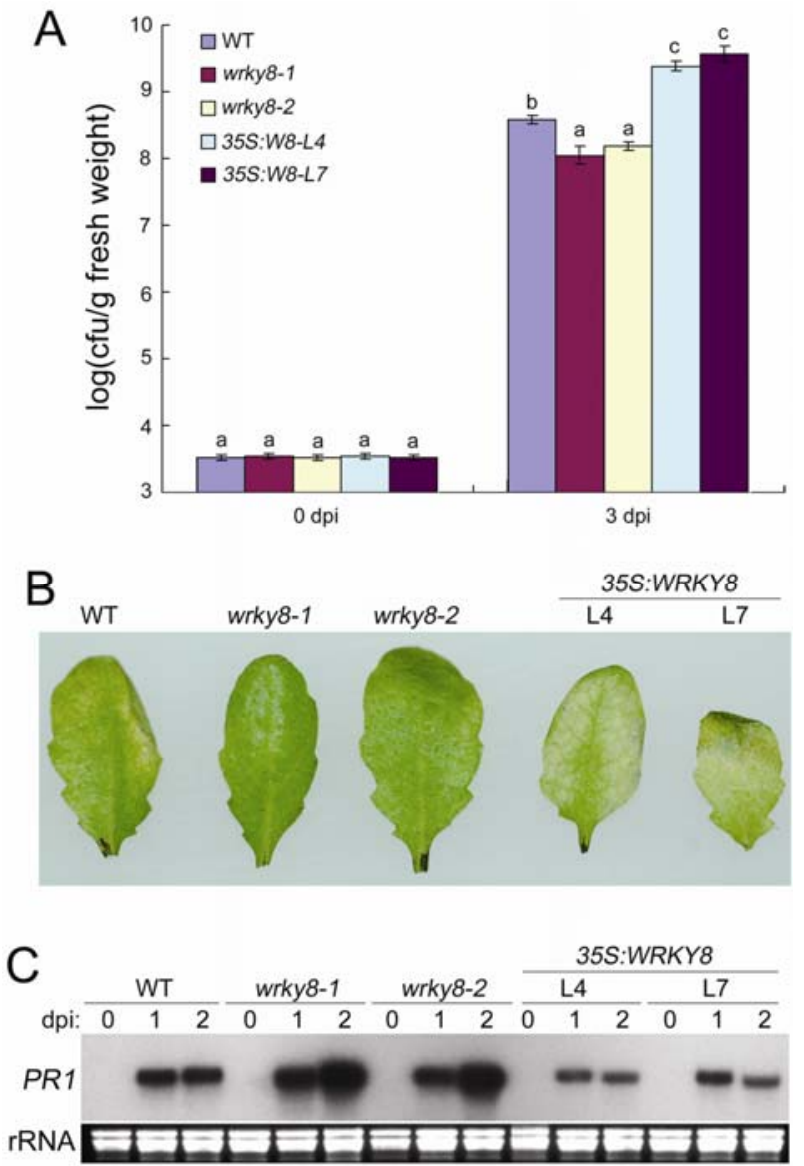

Fig. 6. Altered responses of T-DNA insertion mutants and overexpression plants to Pseudomonas syringae pv. tomato DC3000. A, Altered bacterial growth. Wild-type (WT) (Col-0), wrky 8 mutants, and WRKY 8 overexpression lines were infiltrated with a suspension of $P$. syringae pv. tomato DC3000 (optical density at $600 \mathrm{~nm}=0.0001$ in $10 \mathrm{mM} \mathrm{MgCl}$ ). Growth of the bacterial pathogen was assessed at 0 and 3 days postinoculation (dpi). Means and standard errors are shown ( $n=6$ to 8 plants per treatment). B, Disease symptom development. Pathogen inoculation of WT (Col-0), wrky 8 mutants, and WRKY 8 overexpression lines was performed as in A. Pictures of representative inoculated leaves taken at $3 \mathrm{dpi}$. C, PRl expression. Pathogen inoculation of WT (Col-0), wrky 8 mutants, and WRKY 8 overexpression lines was performed as in A. Total RNAs were isolated from inoculated leaves at 0,1 , and 2 dpi and probed with a PRI fragment. Ethidium bromide-stained ribosomal RNA was used as a loading control. The experiments were repeated twice with similar results. 


\section{Disrupted or altered WRKY8 expression affected the response to $B$. cinerea.}

To determine whether wrky 8 mutant plants are also resistant to a necrotrophic fungal pathogen, we challenged wild-type and wrky 8 mutant plants with $B$. cinerea. Five-week-old plants were inoculated with a $B$. cinerea spore suspension at a density of $5 \times 10^{4}$ spores $/ \mathrm{ml}$. At $6 \mathrm{dpi}$, leaves showing necrotic symptoms were evaluated for disease severity. B. cinerea infection caused necrotic symptoms but necrosis remained at localized sites in the wild-type (Col-0) plants (Fig. 7A). Only $20 \%$ of the leaves of wild-type plants had disease symptoms at 6 dpi (Fig. 7A and B). However, necrotic symptoms rapidly

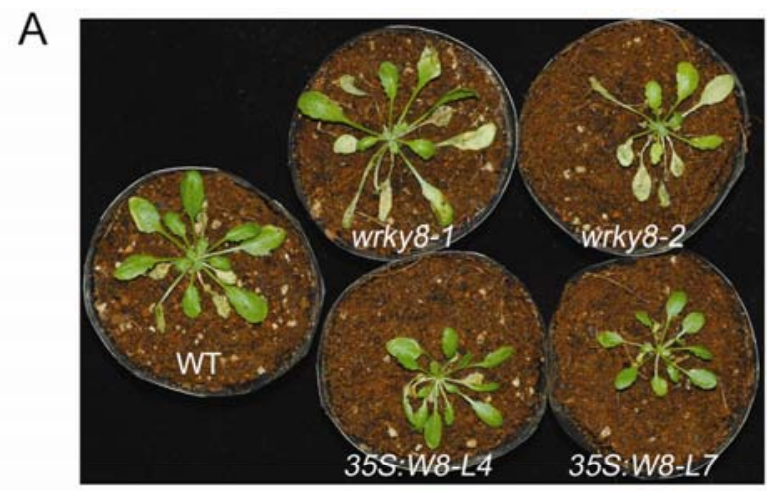

B
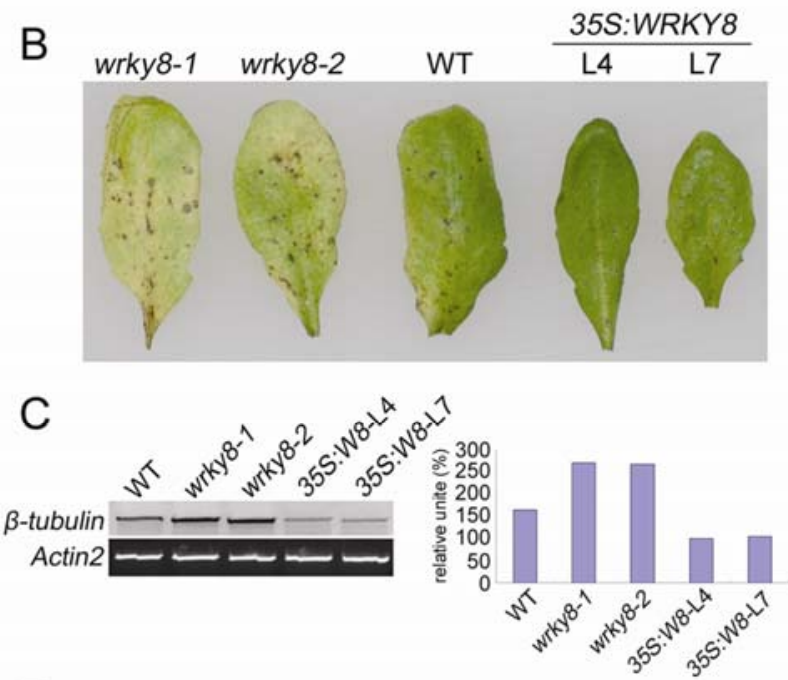

$\mathrm{D}$

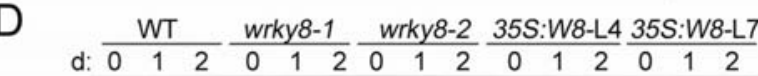

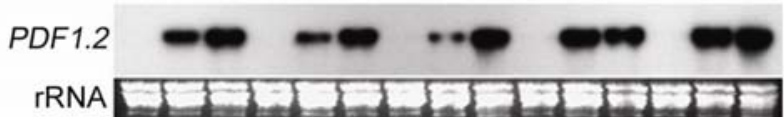

Fig. 7. Responses of T-DNA insertion mutants and WRKY 8 overexpression lines to Botrytis cinerea infection. A and B, Disease symptom development. Leaves of wild-type (WT) (Col-0), wrky 8 mutants, and WRKY 8 overexpression lines were inoculated by spraying with a spore suspension $\left(5 \times 10^{4}\right.$ spores $\left./ \mathrm{ml}\right)$. Plants were maintained at high humidity and disease symptoms were photographed at 6 days postinoculation (dpi). C, Accumulation of $\beta$-tubulin. Total RNA was isolated from inoculated plants at $4 \mathrm{dpi}$ and reverse-transcription polymerase chain reaction was performed with Botrytis spp. $\beta$-tubulin gene-specific primers. Quantification of Botrytis spp. $\beta$-tubulin messenger RNA enabled measurement of fungal biomass on infected plants. The accumulation of various transcripts was quantified by densitometry using Bio-Profile Bio1D software. D, PDF1.2 expression. Pathogen inoculation of WT (Col-0), mutants, and overexpression lines was performed as in A. Total RNAs were isolated from inoculated leaves harvested at 0,1 , and 2 dpi and probed with a PDF1.2 fragment. Ethidium bromide-stained ribosomal RNA was used as a loading control. The experiments were repeated twice with similar results. increased in severity during infection in wrky 8 mutant plants, and approximately $50 \%$ of the leaves were severely decayed at 6 dpi (Fig. 7A and B). In addition, higher levels of $\beta$-tubulin mRNA of $B$. cinerea had accumulated at 4 dpi (Fig. 7C). Thus, disruption of WRKY8 slightly enhanced susceptibility to the fungal pathogen.

To further characterize the role of WRKY 8 in defense against the fungal pathogen, we compared pathogen growth in $35 S:$ WRKY 8 transgenic plants with that in wild-type plants. Conversely to wrky 8 mutants, only $10 \%$ of the leaves of 35S: WRKY8 transgenic plants had disease symptoms at $6 \mathrm{dpi}$, and lower levels of $\beta$-tubulin mRNA of $B$. cinerea accumulated by 4 dpi (Fig. 7A, B, and C). Thus, constitutive overexpression of WRKY8 enhanced tolerance toward the fungal pathogen $B$. cinerea and decreased development of disease symptoms in the transgenic plants.

\section{Expression of defense-related genes.}

Plants with mutations or constitutive overexpression of WRKY8 showed contrasting phenotypes with respect to $P$. syringae and $B$. cinerea resistance. This may reflect an antagonistic relationship between SA- and JA/ET-regulated defense pathways. To test this possibility, we analyzed pathogen-induced expression of SA-regulated PRI and JA-regulated PDF1.2 in both loss-of-function and overexpressing transgenic plants (Glazebrook 2005). After inoculation with $P$. syringae, wrky 8 mutants showed enhanced expression of the $P R l$ gene (Fig. 6C). Transgenic plants constitutively overexpressing WRKY8 showed decreased expression of $P R I$ at 1 and 2 dpi compared with that in wild-type plants. In contrast, after Botrytis spp. infection, there were lower levels of PDF1.2 transcripts in wrky 8 mutants and higher levels of PDF1.2 transcripts in WRKY8-overexpressing lines when compared with the wild-type plants, especially at 1 dpi (Fig. 7D).

\section{DISCUSSION}

Arabidopsis WRKY8 is a stress- and pathogen-induced WRKY gene that encodes a nuclear localized WRKY transcriptional factor. To determine its roles in plant defense against microbial pathogens, we analyzed both knockout lines and constitutive overexpression lines with respect to pathogen infection. Two pathogens were tested: the bacterial pathogen $P$. syringae and the fungal pathogen $B$. cinerea.

$P$. syringae is a biotrophic bacterial pathogen in the early stages of infection but can become necrotrophic at later stages of infection. Thus, it should probably be considered to be a hemibiotroph. Numerous studies have demonstrated that SAmediated signaling mechanisms play vital roles in limiting $P$. syringae growth. For example, Arabidopsis mutants defective in SA biosynthesis or signaling, such as eds 1, pad4, eds5, sid2, and $n p r l$, allow increased growth of $P$. syringae (Glazebrook et al. 1996; Rogers and Ausubel 1997; Aarts et al. 1998; Zhou et al. 1998; Nawrath and Metraux 1999). Mutants with constitutively high SA levels, such as accelerated cell death 6 (acd6) and aberrant growth and death 2 (agd2) (Rate et al. 1999; Rate and Greenberg 2001), displayed increased resistance to $P$. syringae. Plants with defective wrky 8 exhibited a two- to threefold reduction in bacterial growth compared with wild-type plants (Fig. 6A). In contrast, constitutive overexpression of WRKY8 led to enhanced susceptibility to the bacterial pathogen $P$. syringae, as manifested by enhanced growth of the bacterial pathogen and increased and more rapid development of disease symptoms (Fig. 6A and B). Similar to WRKY18 and WRKY48 (Chen and Chen 2002; Xing et al. 2008), overexpression of WRKY8 resulted in transgenic plants that were smaller in size and showed a slightly higher degree of serration in the leaves (Fig. 5D). 
However, WRKY 8 and WRKY48 have effects opposite to that of WRKY18 with respect to defense against $P$. syringae. Thus, we can deduce that the more susceptible phenotype of the transgenic 35S:WRKY8 plants are unlikely to result from their altered growth and morphology. In addition, we observed that the enhanced resistance or susceptibility of wrky 8 mutant plants and transgenic plants to $P$. syringae was associated with increased or reduced expression of $P R l$, a defense-related gene often associated with SA-mediated defense responses. Thus, WRKY 8 might play a negative role in SA-mediated signaling pathways. Overexpression of the gene could have a negative impact on pathogen-induced, SA-mediated defense mechanisms and, thus, compromise plant resistance to $P$. syringae.

B. cinerea is a necrotrophic fungal pathogen that promotes host cell death at very early stages in infection. In Arabidopsis, resistance to $B$. cinerea depends on JA and ET signaling pathways because mutations that block JA signaling, including coil and jarl, or ET signaling, including ein2, result in enhanced susceptibility (Glazebrook 2005). Our analyses revealed that loss-of-function mutants for the WRKY8 gene were slightly more susceptible to $B$. cinerea than the wild-type plants, as measured by enhanced disease symptoms and increased pathogen growth in inoculated plants (Fig. 7A and B). On the other hand, constitutive overexpression of WRKY8 led to slightly decreased susceptibility to the fungal pathogen. Interestingly, these altered phenotypes were opposite to those observed with the bacterial pathogen $P$. syringae. Furthermore, expression of $P D F 1.2$, a molecular marker of the JA- and ET-mediated defense response signaling pathways, differed between the two transgenic lines. Expression of PDF1.2 was decreased in wrky 8 mutants after Botrytis spp. infection but increased in WRKY8-overexpressing transgenic plants.

Our results show that wrky 8 mutants and WRKY8-overexpressing transgenic plants showed opposite responses with respect to pathogen-induced expression of SA-induced $P R 1$ and JA-regulated PDF1.2 (Glazebrook 2005). These opposite responses to the two pathogens probably reflect the antagonism between SA- and JA-mediated defense signaling pathways (Kunkel and Brooks 2002; Li et al. 2004; Takahashi et al. 2004; Pieterse et al. 2009). Together, our results suggest that the WRKY8 protein may function as a negative regulator in SA-dependent pathways but as a positive regulator in JA-mediated pathways. The expression of SID2, NPR1, JAR1, or JAZ1 were not affected in WRKY8-overexpressing or mutant plants, which suggested that $W R K Y 8$ may work at a point downstream of both SA and JA to repress or activate defense responses (Supplementary Fig. 3). Similarly to WRKY8, WRKY33 functions as a positive regulator in JA-mediated defense response signaling and also acts as a repressor of SA-dependent disease resistance (Andreasson et al. 2005; Zheng et al. 2006). In contrast, WRKY70 plays a positive role in SA signaling and functions as a negative regulator of JA-inducible genes $(\mathrm{Li}$ et al. 2004). These examples support the existing trade-off between SA-dependent and JA-dependent defense against biotrophic and necrotrophic pathogens.

We carried out expression analyses using the GUS reporter gene fused to the Arabidopsis WRKY8 gene promoter. Our results show that this gene is expressed mainly in vascular bundles in response to $\mathrm{H}_{2} \mathrm{O}_{2}$, wounding, or rubbing treatments. This suggests that reactive oxygen species (ROS) may be candidates as signaling molecules that mediate pathogen-induced expression of the WRKY 8 gene. Pathogen infection results in generation of ROS and causes oxidative stress in plants (Lamb and Dixon 1997). The induction of WRKY8 through ROS-mediated signaling mechanisms is also consistent with its demonstrated role in defense against hemibiotrophic or necrotrophic pathogens. These pathogens promote ROS generation, which can contribute to induction of cell death. The preferential expression of WRKY in the vascular bundles might contribute to plant defense because these tissues may be important for systemic defense signaling.

Several previous studies have suggested that different WRKY proteins played distinct roles in various signaling pathways of plant defense responses. Interestingly, most of the reported WRKY transcriptional factors, such as WRKY7, WRKY11, WRKY17, WRKY25, WRKY48, WRKY38, and WRKY62, function as negative regulators of plant basal defense against $P$. syringae (Park et al. 2005; Journot-Catalino et al. 2006; Kim et al. 2006, 2008; Zheng et al. 2007, Xing et al. 2008). At the same time, the W-box sequences upstream of the PRl gene promoter act as a negative cis-acting element in the expression of the defense-related gene (Lebel et al. 1998). This implied that defense genes, such as $P R 1$, can be directly repressed by certain WRKY proteins. By contrast, some WRKY transcriptional factors, such as WRKY4 and WRKY33, play positive roles in the basal defense against Botrytis spp. (Zheng et al. 2006; Lai et al. 2008). The diverse roles of WRKY proteins may reflect the complex signaling and transcriptional networks of plant defense that require tight regulation and fine-tuning. The underlying mechanisms for the antagonistic interactions between SA-dependent and JA-dependent defense signaling have not been clearly understood. Identification of WRKY transcription factors that affect plant resistance in ways opposite to different types of microbial pathogens suggest that the regulation of the crosstalk between these defense-signaling pathways occurs at the transcription level and also contribute to further elucidate the mechanisms of the antagonistic actions between defense-response pathways.

\section{MATERIALS AND METHODS}

\section{Materials.}

We obtained $\left[{ }^{32} \mathrm{P}\right]-\mathrm{dATP}(>3,000 \mathrm{Ci} / \mathrm{mmol})$ from the Beijing Furui Biotechnology Co., Ltd. ABA, SA, MeJA, ACC, and 5bromo-4-chloro-3-indolyl b-D-glucuronic acid (X-gluc) were purchased from Sigma Co. Ltd. (St. Louis); Taq DNA polymerase from TaKaRa Biotechnology (Dalian) Co. Ltd. (Tokyo); and agarose and agar from Shanghai Sangon Biotechnology Co. Ltd. (Shanghai, China) Arabidopsis thaliana plants were grown in an artificial growth chamber at $22^{\circ} \mathrm{C}$ under $180 \mu \mathrm{E}$ $\mathrm{m}^{2} \mathrm{~s}^{-1}$ light with a photoperiod of $10 \mathrm{~h}$ of light and $14 \mathrm{~h}$ of darkness. Columbia-0 (Col) was used as the wild type. We obtained wrky 8 mutants in the Col background from the Arabidopsis Biological Resource Center.

\section{Induction treatments.}

SA was dissolved in water as a $100-\mathrm{mM}$ stock solution and adjusted to $\mathrm{pH} 6.5$ with $\mathrm{KOH}$. Plants were sprayed with a 2-mM SA solution diluted from the stock. MeJA was dissolved in $50 \%$ ethanol as a $10-\mathrm{mM}$ stock solution. The MeJA stock solution was diluted to $100 \mu \mathrm{M}$ with water and sprayed onto plants. ACC was dissolved in water, and a 2-mM solution was sprayed onto plants. ABA (14.1 mg) was first dissolved in $90 \mu \mathrm{l}$ of ethanol and then water was added to obtain a $10-\mathrm{mM}$ stock solution. The ABA stock solution was diluted to $100 \mu \mathrm{M}$ with water and sprayed onto plants. $\mathrm{H}_{2} \mathrm{O}_{2}$ was diluted with water and a 5-mM solution was sprayed onto plants. Wound lesions were generated with forceps by squeezing rosette leaves two to three times, which wounded approximately $50 \%$ of the leaf area. In all cases, the aerial parts of 4-week-old plants grown in soil were used.

\section{Subcellular localization.}

WRKY8 cDNA was cloned into a GFP vector and subcloned into the Agrobacterium spp. transformation vector pOCA30 
(Chen and Chen 2002) in the sense orientation behind the CaMV 35S promoter. The construct was then transformed into Agrobacterium tumefaciens GV3101. For transient expression in $N$. benthamiana, leaves of wild-type $N$. benthamiana were infiltrated with the bacterial cell suspensions (optical density at $600 \mathrm{~nm}\left[\mathrm{OD}_{600}\right]=0.05,10 \mathrm{mM} \mathrm{MES}, 10 \mathrm{mM} \mathrm{MgCl}_{2}$, and $100 \mathrm{mM}$ acetosyringone). Leaves were sectioned 24 to $48 \mathrm{~h}$ after infiltration and localization was observed under a confocal laser scanning microscope (Zeiss, Oberkochen, Germany).

\section{Northern blotting and RT-PCR analysis.}

For Northern blotting analyses, total RNA was extracted using TRIzol reagent (Invitrogen, Carlsbad, CA, U.S.A.). Approximately $20 \mu \mathrm{g}$ of RNA was separated on an agaroseformaldehyde gel and then blotted onto nylon membranes following standard procedures. The membranes were hybridized with $\left(\alpha-{ }^{32} \mathrm{P}\right)$-dATP-labeled DNA probes. Hybridization was performed in PerfectHyb Plus hybridization buffer (Sigma) for $16 \mathrm{~h}$ at $68^{\circ} \mathrm{C}$. The membranes were washed once for $10 \mathrm{~min}$ with $2 \times \mathrm{SSC}(1 \times \mathrm{SSC}$ is $0.15 \mathrm{M} \mathrm{NaCl}$ plus $0.015 \mathrm{M}$ sodium citrate) and $0.5 \%$ sodium dodecyl sulfate (SDS), twice for $20 \mathrm{~min}$ with $0.5 \times \mathrm{SSC}$ and $0.1 \%$ SDS, once for $20 \mathrm{~min}$ with $0.1 \times \mathrm{SSC}$ and $0.1 \% \mathrm{SDS}$ at $68^{\circ} \mathrm{C}$, and then exposed to $\mathrm{X}$ ray films at $-80^{\circ} \mathrm{C}$. DNA probes for WRKY8 were isolated from its full-length cDNA clone. DNA probes for $P R I$ and PDF1.2 were obtained from PCR amplifications using the following gene-specific primers: $P R 1,5^{\prime}$-TCTTCCCTCGAA AGCTCAAG-3' and 5'-ACACCTCACTTTGGCACATC-3'; and $P D F 1.2,5^{\prime}$-ACGGGAAGATGATGTCTGTTT-3' and 5' TTCAGTGGTCCTGTTGTAGACA-3'.

For RT-PCR analysis, total RNA was extracted using TRIzol reagent (Invitrogen) and was treated with RNase-free DNase (Fermentas, Vilnius, Lithuania) according to the manufacturer's instructions. Total RNA $(2 \mu \mathrm{g})$ was reverse-transcribed in a $20-\mu$ reaction mixture using the Superscript II (Invitrogen). After the reaction, 1- $\mu$ l aliquots were used as a template for PCR amplification with the following primers: $5^{\prime}$ ATGATCTCTTCCGTGTGCCA-3' and 5'-ATCATCAAGGC TCTTGTTTGAAGA-3' for WRKY8, 5'-TGTGCCAATCTAC GAGGGTTT-3' and 5'-TTTCCCGCTCTGCTGTTGT-3' for ACTIN2, 5'-AGCCTACGATCAAGCTGCTTT-3' and 5'-TTC ACCAAGTCCCACTATTTTCA-3' for ERF1, 5'-CCATTGA CAGAACTTCCTATTGCT-3' and 5'-GGTTAGCATTCATA TTTCAGCTGC-3' for JAZ1, 5'-AAGACTATTGGAGCCTT GGAGC-3' and 5'-AGTGAGTCAAAACGCTGTGCT-3' for $J A R 1,5^{\prime}$-AATTTGCAGTCGGGATCAGAT-3' and 5'-ACTC TTTCTTCAATTAATCGCCTGT-3' for SID2, 5'-ACACTAA AGAAGGCCTTTAGTGAGGA-3' and 5'-TAAGAGGCAAG AGTCTCACCGA-3' for NPR1.

PCR amplification was then performed with Taq DNA polymerase (TaKaRa-Bio).

\section{Isolation of wrky 8 T-DNA insertion mutants.}

The wrky8-1 (SALK_107668), wrky8-2 (SALK_ 050194), and wrky8-3 (SALK_117175) lines contain a T-DNA insertion in the first intron, second intron, and third exon, respectively, of the WRKY8 gene. We confirmed the T-DNA insertions by PCR using a combination of a gene-specific primer and a TDNA border primer. Homozygous wrky 8 mutant plants were identified by PCR using a pair of primers corresponding to sequences flanking the T-DNA insertion sites (pW8-A, 5'-GG AGAAGATGATCAACGCTCT-3' and pW8-B, 5'-TCTTAAG GTGTAGTCTGCCAAAA-3' for wrky8-1; and pW8-C, 5'-AA GTCGAAGGGACTCCATATTT-3' and pW8-D, 5'-CCATTCA TCATGATATGGACTCT-3' for wrky8-2 and wrky8-3). wrky 8 T-DNA insertion mutants were further confirmed by Northern blot analysis and RT-PCR.
Construction of transgenic overexpression lines.

To generate the $35 S: W R K Y 8$ construct, the cDNA fragment containing the full coding sequence and $3^{\prime}$ untranslated region of WRKY 8 was excised from a cloning plasmid and subcloned into the same restriction sites of the Agrobacterium transformation vector pOCA30 (Chen and Chen 2002) in the sense orientation behind the CaMV $35 \mathrm{~S}$ promoter. Arabidopsis transformation was performed by the floral dip procedure (Clough and Bent 1998). The seed were collected from the infiltrated plants and selected on half-strength Murashige Skoog medium containing kanamycin at $50 \mu \mathrm{g} / \mathrm{ml}$. Kanamycin-resistant plants were transferred to soil 8 days after germination and were grown in a growth chamber.

\section{GUS staining.}

Histochemical detection of GUS activity was performed with X-gluc as the substrate. Plant tissues were first prefixed in ice-cold $90 \%$ ( $\mathrm{vol} / \mathrm{vol}$ ) acetone for $20 \mathrm{~min}$, then washed three times with GUS staining buffer (without X-gluc) before incubation in X-gluc solution (1 mM X-Gluc, $50 \mathrm{mM} \mathrm{NaPO}$ [pH 7], $1 \mathrm{mM} \mathrm{K}_{3} \mathrm{Fe}(\mathrm{CN})_{6}, 1 \mathrm{mM} \mathrm{K} \mathrm{K}_{4} \mathrm{Fe}(\mathrm{CN})_{6}$, and $0.05 \%$ Triton $\mathrm{X}-100)$ under vacuum for $10 \mathrm{~min}$ at room temperature, then incubated overnight at $37^{\circ} \mathrm{C}$. Chlorophyll was removed using several changes of $70 \%$ ethanol; then, tissues were photographed.

\section{Pathogen infection.}

For each treatment, leaves of six to eight plants were inoculated by infiltration with the Pseudomonas syringae pv. tomato DC3000 strain containing the pVSP61 kanamycin-resistant empty plasmid vector $\left.\left(\mathrm{OD}_{600}=0.0001 \text { in } 10 \mathrm{mM} \mathrm{MgCl}\right)_{2}\right)$. Inoculated leaves were harvested $3 \mathrm{dpi}$ and homogenized in 10 $\mathrm{mM} \mathrm{MgCl} 2$. Diluted leaf extracts were plated on King's B medium supplemented with rifampicin $(100 \mu \mathrm{g} / \mathrm{ml})$ and kanamycin $(25 \mu \mathrm{g} / \mathrm{ml})$ and incubated at $25^{\circ} \mathrm{C}$ for 2 days before counting CFU.

$B$. cinerea was grown on $2 \times \mathrm{V} 8$ agar as described previously (Mengiste et al. 2003). To infect plants, conidia were collected from a 10-day-old culture and the spore density was adjusted in Sabouraud Maltose Broth and sprayed using a Preval sprayer. Inoculated plants were maintained at high humidity with a transparent cover in a growth chamber, and symptom development was observed from 5 to $7 \mathrm{dpi}$. Biomass of the fungal pathogen was quantified by RT-PCR of total RNA isolated from inoculated plants. For GUS staining, a single $2.5-\mu \mathrm{l}$ drop of a suspension of $2 \times 10^{5}$ spores $\mathrm{ml}^{-1}$ in Sabouraud maltose broth (SMB) buffer was placed on each leaf.

\section{ACKNOWLEDGMENTS}

We thank the Arabidopsis Resource Center at the Ohio State University for the wrky 8 T-DNA insertion mutants, Z. Chen (Department of Botany and Plant Pathology, Purdue University, West Lafayette, IN, U.S.A.) for Arabidopsis mutants (npr1-3, sid2-3, coil-1, and ein2-1), and anonymous reviewers for insightful comments on early manuscript drafts. This work was supported by the Natural Science Foundation of China (30771223 and 90817003) and the Science Foundation of the Chinese Academy of Sciences (KSCX2-YW-N-007).

\section{LITERATURE CITED}

Aarts, N., Metz, M., Holub, E., Staskawicz, B. J., Daniels, M. J., and Parker, J. E. 1998. Different requirements for EDS1 and NDR1 by disease resistance genes define at least two $\mathrm{R}$ gene-mediated signaling pathways in Arabidopsis. Proc. Natl. Acad. Sci. U.S.A. 95:1030610311.

AbuQamar, S., Chen, X., Dhawan, R., Bluhm, B., Salmeron, J., Lam, S., Dietrich, R. A., and Mengiste, T. 2006. Expression profiling and mutant analysis reveals complex regulatory networks involved in Arabidopsis 
response to Botrytis infection. Plant J. 48:28-44.

Alonso, J. M., Hirayama, T., Roman, G., Nourizadeh, S., and Ecker, J. R. 1999. EIN2, a bifunctional transducer of ethylene and stress responses in Arabidopsis. Science 284:2148-2152.

Alonso, J. M., Stepanova, A. N., Leisse, T. J., Kim, C. J., Chen, H., Shinn, P., Stevenson, D. K., Zimmerman, J., Barajas, P., Cheuk, R., Gadrinab, C., Heller, C., Jeske, A., Koesema, E., Meyers, C. C., Parker, H., Prednis, L., Ansari, Y., Choy, N., Deen, H., Geralt, M., Hazari, N., Hom, E., Karnes, M., Mulholland, C., Ndubaku, R., Schmidt, I., Guzman, P., Aguilar-Henonin, L., Schmid, M., Weigel, D., Carter, D. E., Marchand, T., Risseeuw, E., Brodgden, D., Zeko, A., Crosby, W. L. Berry, C. C., and Ecker, J. R. 2003. Genomewide insertional mutagenesis of Arabidopsis thaliana. Science 301:653-657.

Andreasson, E., Jenkins, T., Brodersen, P., Thorgrimsen, S., Petersen, N. H T., Zhu, S. J., Qiu, J. L., Micheelsen, P., Rocher, A., Petersen, M., Newman, M. A., Nielsen, H. B., Hirt, H., Somssich, I., Mattsson, O., and Mundy, J. 2005. The MAP kinase substrate MKS1 is a regulator of plant defense responses. EMBO (Eur. Mol. Biol. Organ.) J. 24:2579-2589.

Asselbergh, B., De Vleesschauwer, D., and Höfte, M. 2008. Global switches and fine-tuning-ABA modulates plant pathogen defense. Mol. Plant-Microbe Interact. 21:709-719.

Cao, H., Glazebrook, J., Clarke, J. D., Volko, S., and Dong, X. 1997. The Arabidopsis NPR1 gene that controls systemic acquired resistance encodes a novel protein containing ankyrin repeats. Cell 88:57-63.

Chen, C. H., and Chen, Z. X. 2002. Potentiation of developmentally regulated plant defense response by AtWRKY18, a pathogen-induced Arabidopsis transcription factor. Plant Physiol. 129:706-716.

Clough, S. J., and Bent, A. F. 1998. Floral dip: A simplified method for Agrobacterium-mediated transformation of Arabidopsis thaliana. Plant J. 16:735-743.

Dong, J. X., Chen, C. H., and Chen, Z. X. 2003. Expression profile of the Arabidopsis WRKY gene superfamily during plant defense response. Plant Mol. Biol. 51:21-37.

Eulgem, T. 2005. Regulation of the Arabidopsis defense transcriptome Trends Plant Sci. 10:71-78.

Eulgem T., Rushton P. J., Robatzek S., and Somssich I. E. 2000. The WRKY superfamily of plant transcription factor. Trends Plant Sci. 5:199-206

Glazebrook, J. 2005. Contrasting mechanisms of defense against biotrophic and necrotrophic pathogens. Annu. Rev. Phytopathol. 43:205-227.

Glazebrook, J., Rogers, E. E., and Ausubel, F. M. 1996. Isolation of Arabidopsis mutants with enhanced disease susceptibility by direct screening. Genetics 143:973-982.

Jing, S. J., Zhou, X., Song, Y., and Yu, D. Q. 2009. Heterologous expression of OsWRKY23 gene enhances pathogen defense and dark-induced leaf senescence in Arabidopsis. Plant Growth Regul. 58:181-190.

Jones, J. D. G., and Dangl, J. L. 2006. The plant immune system. Nature 444:323-329.

Journot-Catalino, N., Somssich, I. E., Roby, D., and Kroj, T. 2006. The transcription factors WRKY11 and WRKY17 act as negative regulators of basal resistance in Arabidopsis thaliana. Plant Cell 18:3289-3302.

Kim, K. C., Fan, B. F., and Chen, Z. X. 2006. Pathogen-induced Arabidopsis WRKY7 is a transcriptional repressor and enhances plant susceptibility to Pseudomonas syringae. Plant Physiol. 142:1180-1192.

Kim, K. C., Lai, Z. B., Fan, B. F., and Chen, Z. X. 2008. Arabidopsis WRKY38 and WRKY62 transcription factors interact with histone deacetylase 19 in basal defense. Plant Cell 20:2357-2371.

Kloek, A. P., Verbsky, M. L., Sharma, S. B., Schoelz, J. E., Vogel, J., Klessig, D. F., and Kunkel, B. N. 2001. Resistance to Pseudomonas syringae conferred by an Arabidopsis thaliana coronatine-insensitive (coi1) mutation occurs through two distinct mechanisms. Plant J. 26:509-522.

Knoth, C., Ringler, J., Dangl, J. L., and Eulgem, T. 2007. Arabidopsis WRKY70 is required for full RPP4-mediated disease resistance and basal defense against Hyaloperonospora parasitica. Mol. PlantMicrobe Interact. 20:120-128.

Kunkel, B. N., and Brooks, D. M. 2002. Cross talk between signaling pathways in pathogen defense. Curr. Opin. Plant Biol. 5:325-331.

Lai, Z. B., Vinod, K., Zheng, Z. Y., Fan, B. F., and Chen, Z. X. 2008. Roles of Arabidopsis WRKY3 and WRKY4 transcription factors in plant responses to pathogens. BMC Plant Biol. 8:68.

Lamb, C., and Dixon, R. A. 1997. The oxidative burst in plant disease resistance. Annu. Rev. Plant Physiol. Plant Mol. Biol. 48:251-275.

Lebel, E., Heifetz, P., Thorne, L., Uknes, S., Ryals, J., and Ward, E. 1998. Functional analysis of regulatory sequences controlling PR-1 gene expression in Arabidopsis. Plant J. 16:223-233.

Li, J., Brader, G., and Palva, E. T. 2004. The WRKY70 transcription factor: A node of convergence for jasmonate-mediated and salicylate-mediated signals in plant defense. Plant Cell 16:319-331.
Li, J., Brader, G., Kariola, T., and Palva, E. T. 2006. WRKY70 modulates the selection of signaling pathways in plant defense. Plant J. 46:477-491.

Liu, Y. L., Schiff, M., and Dinesh-Kumar, S. P. 2004. Involvement of MEK1 MAPKK, NTF6 MAPK, WRKY/MYB transcription factors, COI1 and CTR1 in N-mediated resistance to Tobacco mosaic virus. Plant J. 38:800-809.

Mauch-Mani, B., and Mauch, F. 2005. The role of abscisic acid in plantpathogen interactions. Curr. Opin. Plant Biol. 8:409-414.

Mengiste, T., Chen, X., Salmeron, J., and Dietrich, R. 2003. The BOTRYTIS SUSCEPTIBLE1 gene encodes an R2R3MYB transcription factor protein that is required for biotic and abiotic stress responses in Arabidopsis. Plant Cell 15:2551-2565.

Nawrath, C., and Metraux, J. P. 1999. Salicylic acid induction-deficient mutants of Arabidopsis express PR-2 and PR-5 and accumulate high levels of camalexin after pathogen inoculation. Plant Cell 11:1393-1404.

Park, C. Y., Lee, J. H., Yoo, J. H., Moon, B. C., Choi, M. S., Kang, Y. H., Lee, S. M., Kim, H. S., Kang, K. Y., Chung, W. S., Lim, C. O., and Cho, M. J. 2005. WRKY group IId transcription factors interact with calmodulin. FEBS (Fed. Eur. Biochem. Soc.) Lett. 579:1545-1550.

Pieterse, C. M., Leon-Reyes, A., Van der Ent, S., and Van Wees, S. C. 2009. Networking by small-molecule hormones in plant immunity. Nat. Chem. Biol. 5:308-316.

Qiu, Y. P., and Yu, D. Q. 2009. Over-expression of the stress-induced OsWRKY45 enhances disease resistance and drought tolerance in Arabidopsis. Environ. Exp. Bot. 65:35-47.

Rate, D. N., and Greenberg, J. T. 2001. The Arabidopsis aberrant growth and death 2 mutant shows resistance to Pseudomonas syringae and reveals a role for NPR1 in suppressing hypersensitive cell death. Plant J. 27:203-211.

Rate, D. N., Cuenca, J. V., Bowman, G. R., Guttman, D. S., and Greenberg, J. T. 1999. The gain-of-function Arabidopsis acd6 mutant reveals novel regulation and function of the salicylic acid signaling pathway in controlling cell death, defenses, and cell growth. Plant Cell 11:16951708

Rezzonico, E., Flury, N., Meins, F., and Beffa, R. 1998. Transcriptional down-regulation by abscisic acid of pathogenesis-related beta-1,3-glucanase genes in tobacco cell cultures. Plant Physiol. 117:585-592.

Rogers, E. E., and Ausubel, F. M. 1997. Arabidopsis enhanced disease susceptibility mutants exhibit enhanced susceptibility to several bacterial pathogens and alterations in PR-1 gene expression. Plant Cell 9:305-316.

Shen, Q. H., Saijo, Y., Mauch, S., Biskup, C., Bieri, S., Keller, B., Seki, H., Ulker, B., Somssich, I. E., and Schulze-Lefert, P. 2007. Nuclear activity of MLA immune receptors links isolate-specific and basal disease-resistance responses. Science 315:1098-1103.

Spoel, S. H., Koornneef, A., Claessens, S. M. C., Korzelius, J. P., Van Pelt, J. A., Mueller, M. J., Buchala, A. J., Metraux, J. P., Brown, R., Kazan, K., Van Loon, L. C., Dong, X. N., and Pieterse, C. M. J. 2003. NPR1 modulates cross-talk between salicylate- and jasmonate-dependent defense pathways through a novel function in the cytosol. Plant Cell 15:760-770.

Takahashi, H., Kanayama, Y., Zheng, M. S., Kusano, T., Hase, S., Ikegami, M., and Shah, J. 2004. Antagonistic interactions between the SA and JA signaling pathways in Arabidopsis modulate expression of defense genes and gene-for-gene resistance to cucumber mosaic virus. Plant Cell Physiol. 45:803-809.

Wildermuth, M. C., Dewdney, J., Wu, G., and Ausubel, F. M. 2001. Isochorismate synthase is required to synthesize salicylic acid for plant defence. Nature 414:562-565.

Xie, D. X., Feys, B. F., James, S., Nieto-Rostro, M., and Turner, J. G. 1998. COI1: An Arabidopsis gene required for jasmonate-regulated defense and fertility. Science 280:1091-1094

Xing, D. H., Lai, Z. B., Zheng, Z. Y., Vinod, K. M., Fan, B. F., and Chen, Z. X. 2008. Stress- and pathogen-induced Arabidopsis WRKY48 is a transcriptional activator that represses plant basal defense. Mol. Plant 1:459-470.

Xu, X. P., Chen, C. H., Fan, B. F., and Chen, Z. X. 2006. Physical and functional interactions between pathogen-induced Arabidopsis WRKY18, WRKY40, and WRKY60 transcription factors. Plant Cell 18:1310-1326.

Zheng, Z. Y., Abu Qamar, S., Chen, Z. X., and Mengiste, T. 2006. Arabidopsis WRKY33 transcription factor is required for resistance to necrotrophic fungal pathogens. Plant J. 48:592-605.

Zheng, Z. Y., Mosher, S. L., Fan, B. F., Klessig, D. F., and Chen, Z. X. 2007. Functional analysis of Arabidopsis WRKY25 transcription factor in plant defense against Pseudomonas syringae. BMC Plant Biol. 7:2.

Zhou, N., Tootle, T. L., Tsui, F., Klessig, D. F., and Glazebrook, J. 1998. PAD4 functions upstream from salicylic acid to control defense responses in Arabidopsis. Plant Cell 10:1021-1030. 